\title{
Evaluation for Core Competence of Private Enterprises in Xuchang City Based on an Improved Dynamic Multiple-Attribute Decision-Making Model
}

\author{
Jian-min Shen, ${ }^{1}$ Yao-guo Dang, ${ }^{1}$ Wei-jie Zhou, ${ }^{2}$ and Xue-mei $\mathrm{Li}^{3}$ \\ ${ }^{1}$ College of Economics and Management, Nanjing University of Aeronautics and Astronautics, Nanjing 211100, China \\ ${ }^{2}$ Business College, Changzhou University, Changzhou 213164, China \\ ${ }^{3}$ School of Economics, Ocean University of China, Qingdao 266100, China \\ Correspondence should be addressed to Xue-mei Li; xuemeili85@163.com
}

Received 28 April 2015; Revised 30 July 2015; Accepted 17 August 2015

Academic Editor: Babak Shotorban

Copyright ( 2015 Jian-min Shen et al. This is an open access article distributed under the Creative Commons Attribution License, which permits unrestricted use, distribution, and reproduction in any medium, provided the original work is properly cited.

\begin{abstract}
Because Deng's grey relational degree is inconspicuous, Deng's relational degree with an exponential function is first presented. Then, we demonstrate that improved Deng's relational degree is more conspicuous than the original model. Then, we construct a multiple-attribute decision-making model, based on improved Deng's relational degree with multiple stages, and a method for determining the weight of the index is also developed. Finally, the core competence of private enterprises in Henan province is analyzed, illustrating the validity and feasibility of the improved model.
\end{abstract}

\section{Introduction}

Literature Review of Grey Relational Analysis. Evaluations and decisions are types of problems that are often encountered in daily life [1-3]. Planning, supplier selection, and business performance and investments must be analyzed to determine the alternatives, enterprises, and products to make decisions. Generally, assessments and decision-making are collectively referred to as the decision theory, clustering, and application.

There are many models and methods of decision-making. During the actual decision-making, methods that require more data are not ideal due to the uncertainty and scarcity of information. Considering that the grey system theory can address system problems with regard to poor information, it has been applied to the decision-making field by many scholars. The use of grey relational theory has been researched extensively in solving decision-making problems. When addressing decision-making problems, existing grey relational analysis (GRA) models form the basis for decisionmaking [4-8]. Various types of GRA models can be selected, depending on practical issues.
GRA is predominantly used with decision-making [912] and evaluation methods [13-16]. Grey relational clustering is used by policymakers in group decision-making problems. Song et al. proposed the decision information aggregation decision model after clustering decision-makers' classes and improved the quality of decision-making [17]. Combining grey relational degree and the relational degree of European association, $\mathrm{Hu}$ et al. constructed the average similarity degree to evaluate schemes with regard to the ideal solution and negative ideal positions, clarifying the decisionmaking process [18]. Based on the essential characteristics of the interval grey number, Chen and Liu defined a new interval grey number deviation degree and developed the grey interval incidence coefficient formula and grey relational degree to establish the projection feature vector method and fuzzy complementary judgment decision models, the validity of which has been proven in many examples [19].

For multiattribute risk decision-making problems with continuous random variables, based on the value of property, Liu and Guan generated a similar decision-making model using the grey relational degree and provided the 
decision-making steps [20]. Introducing the punished variable weight, Yang and Chen constructed a grey relational decision model, based on the variable weight and TOPSIS, and applied it to project bidding, and their results demonstrate that the new method is valid and stable [21].

For multiple-attribute decision-making problems with intuitionist fuzzy numbers, Wei determined the weight using the grey relational degree. The calculation steps of decision information aggregation are based on positive, negative, and ideal degrees. A numerical example has been given to illustrate the effectiveness and value of this proposed approach [22]. Based on the evaluation model of the grey relational degree, Lee and Lin assessed energy consumption in office buildings [23]. Combining the VIKOR algorithm and grey combination, Kuo and Liang introduced a fuzzy multiattribute decision-making model to examine service quality problems of international airlines. This decision model is effective and practical in assessing quality of service [24]. Using the fuzzy analytical hierarchy process and grey relational degree, Samvedi et al. constructed the multiattribute decision-making model for machine tool selection. Compared with other machine selection decision models, this model performs better [25].

The GRA model has been widely applied to societal economics and engineering practice problems, such as in determining regional economy development gaps [26], identifying the factors that influence service brand equity [27], tuning PID control parameters for micro-piezo-stage [28], examining utilization [29], establishing laws of maximum flood peak in the upstream Yellow River [30], and failure mode and effects analysis [31].

Literature Review of Evaluation of Enterprises' Core Competence. With over 30 years of reform and opening-up of market, Chinese private enterprises have achieved rapid development and have gradually become an important part of the socialist market economy and a new source of growth for the national economy. During the development of China's private enterprises, their core competitive ability has undergone constant evolution and development. Scholars have examined evaluation models of enterprise competence and their applications in specific industries.

The integrity, systematics, externality, and timeliness of evaluation methods have garnered the most attention, and, despite many studies on the evaluation of enterprises' core competence from a knowledge and finance perspective, the literature on the core competence of enterprises with regard to the entire operation continues to grow. Feng and Yi developed an evaluation index system for financial structure, scale strength, profitability, operational capacity, and development, based on the sum and absorption of the classical financial evaluation of core competence [32]. Xia extracted the explicit evaluation index for the capability, resources, and survival of enterprises by analyzing the top 20 small and mediumsized enterprises on the Shenzhen Stock Exchange and built an overall appraisal model of competitiveness for them [33].

Feng and Diao decomposed profit, operation, scale, and growth indices into 13 second-level indexes by principal component analysis and evaluated the core competitiveness of life insurance enterprises, considering time as a factor, after defining the scope of their competitiveness [34]. Combined with the sustainable development theory and competitive advantage theory, Wang et al. developed an index to evaluate enterprise competitiveness with regard to resource conditions, the structure and benefit of the industry, development and support, and sustainable effects [35].

The evaluation index has changed from qualitative and quantitative or semiquantitative evaluation to a nearly quantitative assessment. Liu et al. combined the AHP and Delphi method and analyzed evaluation indexes of 4PL: market competition ability, core business ability, information technology, organizational ability, management, and local government capacity [36].

Research on evaluation models has shifted from finance, energy, and innovation to examples of enterprises in culture, education, logistics, medicine, automobile, and other industries. Further, more attention has been paid to the differences in establishing evaluation index systems between industries, prompting the development of models to evaluate industries and regions. Wang divided evaluation indexes of headquarters' economy into 3 levels. The first level comprises the basic conditions, business development, service support, and degree of openness [37]. Cai presented 4 evaluation indexes, industry input, industry output, industry market performance, and industry technical level, using principal component analysis and evaluated the competitiveness of Xiamen's 29 manufacturing industries [38].

Zhang learned from theories on enterprise competitiveness and studied methods and index systems to evaluate industry competitiveness of microenterprises, considering the characteristics and competitiveness of small and medium-sized enterprises in various provinces and cities [39]. Yang and Zhu applied the interval fuzzy number method to evaluate the competitiveness of enterprises in culture, education, logistics, medicine, and the iron and steel industries [40]. Chang assessed the core competitiveness of airlines, third-party logistics companies, and logistics companies using the AHP-TOPSIS model [41].

Research Motivation. Based on the literature review above, the grey relational theory has been widely applied to decisionmaking problems. With regard to grey theory decisions, Deng's relational degree is the most popular method. Obviously, for decision-related problems, greater precision in selecting the project or supplier improves the results, which can increase the quality of the decision-making. However, in practical applications, the current grey Deng's relational degree is suboptimal in computing the correlation between indices (e.g., the relevance index distinction) [42], because current Deng's grey relational degree has a lower limit, which is not conducive to identifying the relevance index as weak. Thus, the concept of a new grey relational degree resolution, based on an exponential function, is defined in this paper, and we demonstrate that the precision of improved grey Deng's relational degree resolution is higher than that of the original model.

In the next section, improved grey Deng's relational degree model is presented, and its advantages are discussed. 
The dynamic multiple-attribute assessment model, based on the improved grey Deng's relational degree model, is developed in Section 3. The model is applied to evaluate the core competence of private enterprises in Xuchang City in Section 4. Section 5 presents the conclusions and future research.

\section{Improved Grey Deng's Relational Degree Model}

Definition 1 (see [43]). Assume that the related behavior sequence in a system is

$$
\begin{gathered}
Z_{0}=\left(z_{0}(1), z_{0}(2), \ldots, z_{0}(n)\right) \\
Z_{1}=\left(z_{1}(1), z_{1}(2), \ldots, z_{1}(n)\right) \\
Z_{2}=\left(z_{2}(1), z_{2}(2), \ldots, z_{2}(n)\right) \\
\vdots \\
Z_{i}=\left(z_{i}(1), z_{i}(2), \ldots, z_{i}(n)\right) \\
\vdots \\
Z_{m}=\left(z_{m}(1), z_{m}(2), \ldots, z_{m}(n)\right),
\end{gathered}
$$

where $\rho \in(0,1)$. Thus

$$
\begin{aligned}
& \gamma\left(z_{0}(k), z_{i}(k)\right) \\
& =\frac{\operatorname{mim}_{i} \operatorname{mim}_{k}\left|z_{0}(k)-z_{i}(k)\right|+\rho \max _{i} \max _{k}\left|z_{0}(k)-z_{i}(k)\right|}{\left|z_{0}(k)-z_{i}(k)\right|+\rho \max _{i} \max _{k}\left|z_{0}(k)-z_{i}(k)\right|}, \\
& \gamma\left(Z_{0}, Z_{i}\right)=\frac{1}{n} \sum_{k=1}^{n} \gamma\left(z_{0}(k), z_{i}(k)\right),
\end{aligned}
$$

where $\gamma\left(Z_{0}, Z_{i}\right)$ is known as Deng's grey relational degree and $\rho$ is the distinguishing coefficient.

Definition 2. Assume that $F(X)$ and $G(X)$ are 2 types of grey relational degrees; if any

$$
\left|\frac{F(X+\Delta X)-F(X)}{\Delta X}\right|>\left|\frac{G(X+\Delta X)-G(X)}{\Delta X}\right|,
$$

then the resolution of $F(X)$ is higher than $G(X)$.

In the use of grey relational analysis correlation for different objects with the same index, when the difference in index values is small, the calculated grey correlation degree of $F$ is easier to use to judge the correlation between behavioral sequences than $G$. We can see that the resolution of grey correlation $F$ is larger than $G$. We analyze traditional Deng's grey relational degree.

First, we present traditional Deng's grey relational formula:

$$
\begin{aligned}
& \gamma\left(z_{0}(k), z_{i}(k)\right)=\frac{\operatorname{mim}_{i} \operatorname{mim}_{k}\left|z_{0}(k)-z_{i}(k)\right|+\rho \max _{i} \max _{k}\left|z_{0}(k)-z_{i}(k)\right|}{\left|z_{0}(k)-z_{i}(k)\right|+\rho \max _{i} \max _{k}\left|z_{0}(k)-z_{i}(k)\right|} \\
& \quad=\frac{1}{1+\left(\left|z_{0}(k)-z_{i}(k)\right|-\operatorname{mim}_{i} \operatorname{mim}_{k}\left|z_{0}(k)-z_{i}(k)\right|\right) /\left(\operatorname{mim}_{i} \operatorname{mim}_{k}\left|z_{0}(k)-z_{i}(k)\right|+\rho \max _{i} \max _{k}\left|z_{0}(k)-z_{i}(k)\right|\right)}
\end{aligned}
$$

Set

$x$

$$
\begin{aligned}
& =\frac{\left|z_{0}(k)-z_{i}(k)\right|-\operatorname{mim}_{i} \operatorname{mim}_{k}\left|z_{0}(k)-z_{i}(k)\right|}{\operatorname{mim}_{i} \operatorname{mim}_{k}\left|z_{0}(k)-z_{i}(k)\right|+\rho \max _{i} \max _{k}\left|z_{0}(k)-z_{i}(k)\right|}, \\
& \gamma_{0 i}=\gamma\left(z_{0}(k), z_{i}(k)\right) .
\end{aligned}
$$

Then,

$$
\gamma_{0 i}=\frac{1}{1+x}
$$

Thus, the denominator of $\gamma_{0 i}$ is linear, indicating that the resolution of $\varepsilon_{0 i}$ is not high for different objects (same index correlation). In addition, we can prove that $\gamma_{0 i} \in(0.333,1]$, where $\rho$ is 0.5 in general. Thus, Deng's grey relational degree cannot differentiate between 2 sequences, the correlation of which does not exist or is close to 0 . This is not consistent with the grey relational degree of the standard. When $x$ is small, $e^{x} \approx 1+x$ and $e^{x}$ has smooth, nonlinear characteristics, and ultimately $e^{x}$ will be used to replace the denominator of $\gamma_{0 i}$ to overcome the disadvantages of traditional Deng's grey relational degree.

Definition 3. Assuming the system behavior sequence as shown in Definition 1, we define

$$
\gamma_{0 i}^{\prime}\left(z_{0}(k), z_{i}(k)\right)=e^{-\left(\left|z_{0}(k)-z_{i}(k)\right|-\operatorname{mim}_{i} \operatorname{mim}_{k}\left|z_{0}(k)-z_{i}(k)\right|\right) /\left(\operatorname{mim}_{i} \operatorname{mim}_{k}\left|z_{0}(k)-z_{i}(k)\right|+\rho \max _{i} \max _{k}\left|z_{0}(k)-z_{i}(k)\right|\right)}
$$




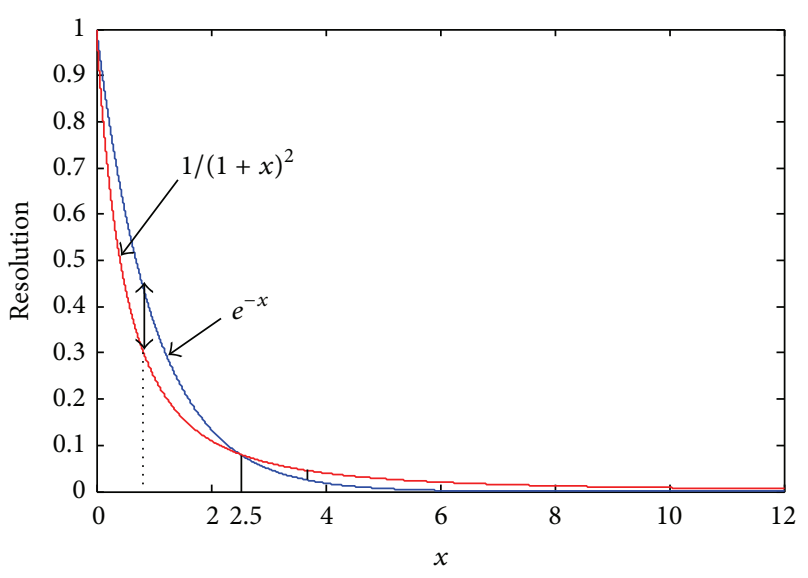

Figure 1: Comparison of the resolution for $e^{-x}$ and $1 /(1+x)$.

as the improved Deng's grey relational degree, where $\rho$ is the resolution coefficient.

Proposition 4. When $x \leq 2.5$, the resolution of the grey relational degree of $\varepsilon_{0 i}^{\prime}=e^{-x}$ is greater than $\varepsilon_{0 i}=1 /(1+x)$.

Proof. Consider the absolute value of the derivative of $e^{-x}$ and $1 /(1+x)$. The absolute values of the derivative $e^{-x}$ and $1 /(1+x)$ are, respectively, $e^{-x}$ and $\varepsilon_{0 i}=1 /(1+x)^{2}$. Because the above conclusion is difficult to prove from the analysis directly, we analyze their size numerically.

From Figure 1, when $x \leq 2.5, e^{-x}>1 /(1+x)^{2}$; that is, the resolution of $\varepsilon_{0 i}^{\prime}$ is greater than $\varepsilon_{0 i}$. When these 2 values are very close, there is no obvious resolution. In addition, improved Deng's grey relational degree avoids the degree with a lower limit. Thus, improved Deng's grey relational degree improves the existing deficiency of Deng's grey relational degree.

\section{Dynamic Multiple-Attribute Assessment Model}

For a multiple-attribute decision-making problem $D$, the evaluation object set $S=\left\{s_{1}, s_{2}, \ldots, s_{n}\right\}$, attribute set $A=$ $\left\{a_{1}, a_{2}, \ldots, a_{m}\right\}$, and stage set $T=\left\{t_{1}, t_{2}, \ldots, t_{p}\right\}$. In the stage $t_{k}$, the attribute value $a_{j}$ of object $s_{i}$ is $r_{i j}^{k} \cdot i=1,2, \ldots, s$, $j=1,2, \ldots, m, k=1,2, \ldots, p$.

The weight of attribute $a_{j}$ in stage $t_{k}$ is set to $\lambda_{j}^{k}$ to evaluate enterprise production, management, and development. We need a general evaluation of the various aspects of the management of each enterprise, necessitating an evaluation model to obtain the overall appraisal of each enterprise value; the key issue is determining how to calculate the weights of enterprises that operate in disparate aspects and stages of development. Because less data can be generated in the assessment of enterprises, we solve business development problems by the grey evaluation system. Grey relational degree is used to determine attribute weights and time weight.
The relationship between system factors is measured, and the superior and inferior system factors are determined, resulting in the weight of each factor. The steps are as follows.

Step 1. Select the reference sequence. With $n(i=1,2, \ldots, n)$ evaluation units (enterprises) and $m(j=1,2, \ldots, m)$ evaluation indicators, $r_{i j}^{k}$ is the evaluation value of the $i$ th evaluation unit of the $j$ th unit in stage $k$; all $r_{i j}^{k}$ values constitute the following matrix:

$$
R^{k}=\left[\begin{array}{cccc}
r_{11}^{k} & r_{12}^{k} & \cdots & r_{1 m}^{k} \\
r_{21}^{k} & r_{22}^{k} & \cdots & r_{2 m}^{k} \\
\cdots & \cdots & \ddots & \cdots \\
r_{n 1}^{k} & r_{n 2}^{k} & \cdots & r_{n m}^{k}
\end{array}\right]
$$

Step 2. Construct the positive ideal solution using

$$
\begin{aligned}
& r^{k+}=\left\{r_{1}^{k+}, r_{2}^{k+}, \ldots, r_{m}^{k+}\right\} \\
& r_{j}^{k+}=\max _{n \leq i \leq n}\left\{r_{i j}^{k}\right\}, \quad j=1,2, \ldots, m .
\end{aligned}
$$

Step 3. Calculate the positive ideal solution of attribute values using grey correlation degree

$$
\begin{aligned}
& \gamma_{i j}^{k+} \\
& =e^{-\left(\left|r_{i j}^{k}-r_{j}^{k+}\right|-\operatorname{mim}_{i} \operatorname{mim}_{j}\left|r_{i j}^{k}-r_{j}^{k+}\right|\right) /\left(\operatorname{mim}_{i} \operatorname{mim}_{j}\left|r_{i j}^{k}-r_{j}^{k+}\right|+\rho \max _{i} \max _{j}\left|r_{i j}^{k}-r_{j}^{k+}\right|\right)},
\end{aligned}
$$

where is 0.5 .

Step 4. Calculate attribute weights by

$$
\begin{aligned}
\lambda_{j}^{k}= & \frac{\sum_{i=1}^{n} \gamma_{i j}^{k+}}{\sum_{j=1}^{m} \sum_{i=1}^{n} \gamma_{i j}^{k+}} \\
& \quad i=1,2, \ldots, n, j=1,2, \ldots, m, k=1,2, \ldots, p .
\end{aligned}
$$

Step 5. Determine the time weight and negative ideal scheme with

$$
\begin{aligned}
& r^{k-}=\left\{r_{1}^{k-}, r_{2}^{k-}, \ldots, r_{m}^{k-}\right\}, \\
& r_{j}^{k-}=\min _{n \leq i \leq n}\left\{r_{i j}^{k}\right\}, \quad j=1,2, \ldots, m .
\end{aligned}
$$

Evaluate objects $S_{i}$ in the stage $k$ whole positive, negative deviation, respectively, as follows:

$$
\begin{aligned}
& D_{i}^{k+}=\sum_{j=1}^{m}\left|r_{i j}^{k}-r_{j}^{k+}\right| \lambda_{j}^{k}, \\
& D_{i}^{k-}=\sum_{j=1}^{m}\left|r_{i j}^{k}-r_{j}^{k-}\right| \lambda_{j}^{k} .
\end{aligned}
$$


The entire positive and negative deviation of evaluation objects $S_{i}$ is, respectively,

$$
\begin{aligned}
& D_{i}^{+}=\sum_{k=1}^{p} \sum_{j=1}^{m}\left|r_{i j}^{k}-r_{j}^{k+}\right| \lambda_{j}^{k} w_{k}, \\
& D_{i}^{-}=\sum_{k=1}^{p} \sum_{j=1}^{m}\left|r_{i j}^{k}-r_{j}^{k-}\right| \lambda_{j}^{k} w_{k} .
\end{aligned}
$$

Accordingly, the entire evaluated objects' positive and negative deviations, respectively, are

$$
\begin{aligned}
& D^{+}=\sum_{i=1}^{n} \sum_{k=1}^{p} \sum_{j=1}^{m}\left|r_{i j}^{k}-r_{j}^{k+}\right| \lambda_{j}^{k} w_{k}, \\
& D^{-}=\sum_{i=1}^{n} \sum_{k=1}^{p} \sum_{j=1}^{m}\left|r_{i j}^{k}-r_{j}^{k-}\right| \lambda_{j}^{k} w_{k} .
\end{aligned}
$$

Step 6. For system evaluation objects, the entire positive and negative evaluation values are

$$
\begin{aligned}
& D_{i}^{+}=\sum_{k=1}^{p} \sum_{j=1}^{m}\left|r_{i j}^{k}-r_{j}^{k+}\right| \lambda_{j}^{k} w_{k}, \\
& D_{i}^{-}=\sum_{k=1}^{p} \sum_{j=1}^{m}\left|r_{i j}^{k}-r_{j}^{k-}\right| \lambda_{j}^{k} w_{k} .
\end{aligned}
$$

Calculate the close degree $d_{i}=D_{i}^{+} /\left(D_{i}^{+}+D_{i}^{-}\right)$and rank the objects by close degree.

Determining the time weight is as follows.

When determining the time weight, we should determine the positive deviation from the ideal minimum amount and the negative deviation from the ideal maximum. Accordingly, we transform it into the following multiobjective programming problem:

$$
\begin{array}{ll}
\min & D^{+}\left(w_{k}\right)=\sum_{i=1}^{n} \sum_{k=1}^{p} \sum_{j=1}^{m}\left|r_{i j}^{k}-r_{j}^{k+}\right| \lambda_{j}^{k} w_{k} \\
\max & D^{-}\left(w_{k}\right)=\sum_{i=1}^{n} \sum_{k=1}^{p} \sum_{j=1}^{m}\left|r_{i j}^{k}-r_{j}^{k-}\right| \lambda_{j}^{k} w_{k} \\
\text { s.t. } \quad \sum_{k=1}^{p} w_{k}=1, \quad w_{k} \geq 0, k=1,2, \ldots, p .
\end{array}
$$

Because the weight has a certain degree of uncertainty for decision systems with incomplete information, we should reduce the uncertainty of the time weight as much as possible. As per entropy definition, we define time weight as follows:

$$
H(w)=-\sum_{k=1}^{p} w_{k} \ln w_{k} .
$$

As per the maximum entropy principle, we equalize the weight as much as possible; then, the maximum entropy can be expressed as

$$
\begin{array}{ll}
\max & H(w)=-\sum_{k=1}^{p} w_{k} \ln w_{k} \\
\text { s.t. } & \sum_{k=1}^{p} w_{k}=1, \quad w_{k} \geq 0, k=1,2, \ldots, p .
\end{array}
$$

Further, we introduce the coordination coefficient. Because the positive ideal and negative ideal deviations of the total amount are independent, the coefficient can be arranged as $\mu$. Taking the 3 goals into account collectively, the coefficient of $H(w)$ is $1-2 \mu$. Combine the 3 optimization objectives above into a single objective minimization problem:

$$
\begin{array}{ll}
\operatorname{mim} & \left\{\mu \sum_{i=1}^{n} \sum_{k=1}^{p} \sum_{j=1}^{m}\left|r_{i j}^{k}-r_{j}^{k+}\right| \lambda_{j}^{k} w_{k}-\mu \sum_{i=1}^{n} \sum_{k=1}^{p} \sum_{j=1}^{m}\left|r_{i j}^{k}-r_{j}^{k-}\right| \lambda_{j}^{k} w_{k}+(1-2 \mu) \sum_{k=1}^{p} w_{k} \ln w_{k}\right\} \\
\text { s.t. } & \sum_{k=1}^{p} w_{k}=1, \quad w_{k} \geq 0, k=1,2, \ldots, p, \\
\operatorname{mim} \quad & \left\{\mu \sum_{i=1}^{n} \sum_{k=1}^{p} \sum_{j=1}^{m}\left|r_{i j}^{k}-r_{j}^{k+}\right| \lambda_{j}^{k} w_{k}-\mu \sum_{i=1}^{n} \sum_{k=1}^{p} \sum_{j=1}^{m}\left|r_{i j}^{k}-r_{j}^{k-}\right| \lambda_{j}^{k} w_{k}+(1-2 \mu) \sum_{k=1}^{p} w_{k} \ln w_{k}\right\} \\
\text { s.t. } \quad \sum_{k=1}^{p} w_{k}=1, \quad w_{k} \geq 0, k=1,2, \ldots, p,
\end{array}
$$


where $0<\mu<0.5$ is the balance coordination coefficient value for the 3 targets, according to the actual situation analysis. In this paper, considering the 3 goals as equally important, let $\mu=1 / 3$.

Construct the Lagrange function:

$$
\begin{aligned}
L\left(w_{k}, \tau\right)= & \mu \sum_{i=1}^{n} \sum_{k=1}^{p} \sum_{j=1}^{m}\left|r_{i j}^{k}-r_{j}^{k+}\right| \lambda_{j}^{k} w_{k} \\
& -\sum_{i=1}^{n} \sum_{k=1}^{p} \sum_{j=1}^{m}\left|r_{i j}^{k}-r_{j}^{k-}\right| \lambda_{j}^{k} w_{k} \\
& +(1-2 \mu) \sum_{k=1}^{p} w_{k} \ln w_{k}-\tau\left(1-\sum_{k=1}^{p} w_{k}\right) .
\end{aligned}
$$

$$
w_{k}=\exp \left\{\frac{\tau+\mu \sum_{i=1}^{n} \sum_{j=1}^{m}\left|r_{i j}^{k}-r_{j}^{k-}\right| \lambda_{j}^{k}-\mu \sum_{i=1}^{n} \sum_{j=1}^{m}\left|r_{i j}^{k}-r_{j}^{k+}\right| \lambda_{j}^{k}}{1-2 \mu}\right\} .
$$

Because $\sum_{k=1}^{p} w_{k}=1$, we get

$$
w_{k}=\frac{\exp \left\{\left(\mu \sum_{i=1}^{n} \sum_{j=1}^{m}\left|r_{i j}^{k}-r_{j}^{k-}\right| \lambda_{j}^{k}-\mu \sum_{i=1}^{n} \sum_{j=1}^{m}\left|r_{i j}^{k}-r_{j}^{k+}\right| \lambda_{j}^{k}\right) /(1-2 \mu)-1\right\}}{\sum_{k=1}^{p} \exp \left\{\left(\mu \sum_{i=1}^{n} \sum_{j=1}^{m}\left|r_{i j}^{k}-r_{j}^{k-}\right| \lambda_{j}^{k}-\mu \sum_{i=1}^{n} \sum_{j=1}^{m}\left|r_{i j}^{k}-r_{j}^{k+}\right| \lambda_{j}^{k}\right) /(1-2 \mu)-1\right\}} .
$$

\section{Case Study}

In this section, we use the dynamic multiattribute decision model above to evaluate the core competence of private enterprises in Xuchang City over the last 4 years.

4.1. Determination of Index System in the Evaluation Questionnaire for the Core Competence of Xuchang Private Enterprises. The questionnaire is designed for each variable. When designing items, existing scales should be cited as much as possible, and new items that are based on research variables are added. Finally, the evaluation indexes of the core competence of private enterprises in Xuchang are constructed, as in Table 1.

(1) Technological capability includes 3 levels of indexes: equipment and technic, the number of invention patents, $\mathrm{R} \& \mathrm{D}$ staff/workers, $\mathrm{R} \& \mathrm{D}$ funds/gross proceeds from sales, and the ability to innovate with university/institute by production-study-research cooperation.

Equipment and technical level indexes are divided into international advanced level, domestic leading level, and industry average level; the number of invention patents is the number of invention patents that the enterprises own; $\mathrm{R} \& \mathrm{D}$ staff is defined as the number of research fellows; $\mathrm{R} \& \mathrm{D}$ funds are the R\&D funds that are invested; and the ability to innovate with university/institute by productionstudy-research cooperation is the average annual output of collaboration.

(2) Management ability consists of 3 levels of indexes: overall labor productivity, enterprise reputation, management quality of the entrepreneur, knowledge quality, and development ability. Overall labor productivity is the gross profit/staff number; enterprise reputation is divided into Xuchang famous, Henan famous, national famous, and international famous.

Management quality of the entrepreneur consists of 3 levels: good, scored 8-10; common, scored 5-7; and poor, scored 1-4. An entrepreneur who makes quick decisions that are always right and can delegate is a good level; the common level refers to those whose decisions, coordination, and other management methods are common; poor level refers to those with poor comprehensive management.

Knowledge quality is evaluated through knowledge of humanities and social sciences and management expertise. Those with overall knowledge are scored 8-10; those with common knowledge are scored 5-7; and those who are ignorant of the knowledge are scored 1-4.

Development ability consists of the ability to seize market opportunity, promote a market, and innovate and is divided 
TABLE 1: Index system in the questionnaire for the evaluation of the core competence of private enterprises.

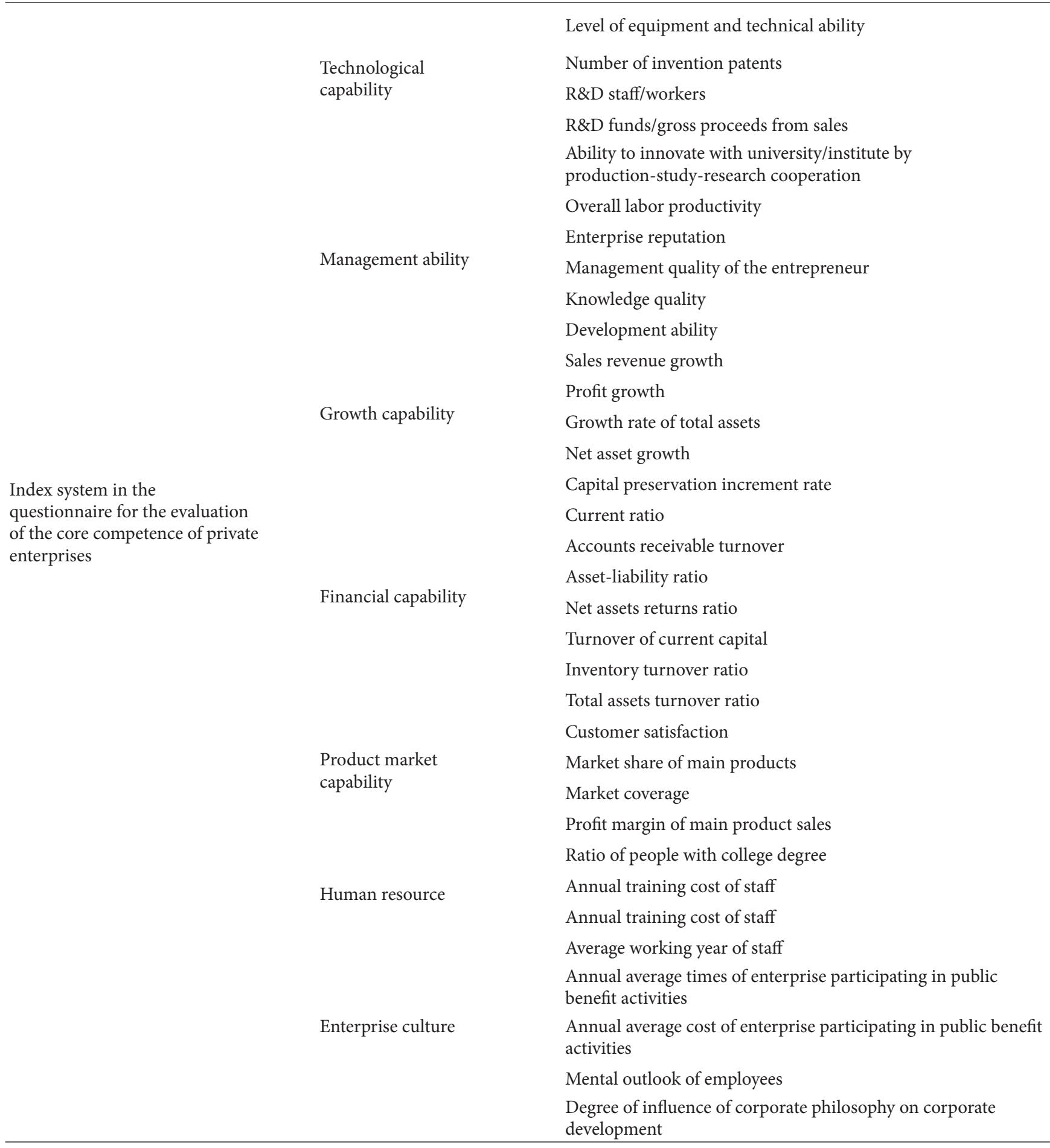

into 3 levels: good, scored 8-10; common, scored 2-4; and poor, scored 0-3.

(3) Growth capability consists of third-level indexes, which are sales revenue growth, profit growth, growth rate of total assets, net asset growth, and capital preservation increment rate.
Sales revenue growth equals the amount of sales revenue growth/original amount of revenue $* 100 \%$.

Profit growth is sales growth in the amount of profit/the amount of original sales profits $* 100 \%$.

Growth rate of total assets is the amount of total asset growth/total amount of the original assets $* 100 \%$. 
Net asset growth is the amount of net asset growth/net amount of the original assets $* 100 \%$.

The increment rate of capital maintenance equals ownership interest at the beginning of year + profits at the end of year/ownership interest at the beginning of year $* 100 \%$.

(4) Financial capability consists of third-level indexes: current ratio, accounts receivable turnover, asset-liability ratio, net assets returns ratio, turnover of current capital, inventory turnover ratio, and total assets turnover ratio.

Current ratio equals total current assets/total current liabilities $* 100 \%$.

Accounts receivable turnover equals net income of credit/average balance of receivables $* 100 \%$.

Asset-liability ratio equals total liabilities/total assets $\times$ $100 \%$.

Net assets returns ratio equals after-tax profits/total net assets $* 100 \%$.

Turnover of current capital equals amount of turnover/average occupancy of current capital $* 100 \%$.

Asset-liability ratio equals cost of goods sold/average balance of stock $* 100 \%$.

Total assets turnover ratio equals net sales/total average assets $* 100 \%$.

(5) Product market capability consists of third-level indexes: customer satisfaction, market share of main products, market coverage, and profit margin of main product sales.

Customer satisfaction is obtained according to the enterprise's survey.

Market share of main products equals market share that equals sales income of enterprise/total sales of market $* 100 \%$.

Market coverage equals the amount of product-launching regions of the enterprise/amount of national product regions.

Profit margin of main products sales equals sales profit of main products/total sales of main products $* 100 \%$.

(6) Human resource consists of third-level indexes: ratio of people with a college degree, annual training cost of staff, annual average training time of staff, and average working year of staff.

The ratio of people with a college degree equals the number of people with a college degree/number of employees.

The annual average training time of the staff is defined as all staff training time in 1 year on average.

The annual training cost of staff refers to all staff training costs in 1 year.

The average working year of the staff equals the total working years of all staff/number of all employees.

(7) Enterprise culture consists of third-level indexes: the annual average times of the enterprise participating in public benefit activities, annual average cost of enterprise participating in public benefit activities, mental outlook of employees, and degree of influence of corporate philosophy on corporate development.

Annual average times of the enterprise participating in public benefit activities equal the total times that the enterprise participates in public benefit activities in the most recent 10 years $/ 10$.
The average cost of an enterprise participating in public benefit activities equals the total cost of the enterprise participating in public benefit activities/10.

The mental outlook of employees is judged by the motivation of the staff to work. An enterprise in which over $80 \%$ of the staff work hard is scored $7-10$; one with over $50 \%$ is scored $4-7$; one with over $20 \%$ is scored 1-3; and those with under $20 \%$ are scored 0 .

The degree of influence of corporate philosophy on corporate development is divided into 3 classes. An enterprise with a philosophy that permeates all aspects and affects its strategic decision-making is given a score of 8-10. In contrast, an enterprise with a philosophy that only has a greater impact in its management scores 4-7 and an enterprise in which its philosophy is merely a slogan is given 0 .

4.2. Calculation and Results of Evaluation. There are 34 indicators on technical ability, management ability, growth ability, financial capacity, product market resources, human resources, and enterprise culture, forming the enterprise's comprehensive ability from 2010 to 2013.

Except for the asset liabilities rate being a moderate index, the rest are profitability indexes. To ensure that the results are not affected by the index dimension, we proceed as follows: profitability index $X_{i j}=A_{i j} / \max _{j} A_{i j},(i=1,2, \ldots, n, j=$ $1,2, \ldots, m)$, and $A_{i j}$ is an index. The results can be seen during $[0,1]$. For the moderate index asset liabilities rate, a $40 \%$ to $60 \%$ rate for the enterprise is favorable; therefore, we choose $50 \%$ as a moderate value, treated as follows: $X_{i j}=$ $1-\left|0.5-A_{i j}\right| / 0.5$. In this paper, $0<A_{i j}<1$; thus, the index after the conversion rate of assets and liabilities remains in the range of $[0,1]$. Based on the attribute weights and time weight method above, we can obtain the results. According to the findings, from 2010 to 2013, the ranking of the four enterprises with regard to comprehensive core competence is as follows: Henan Senyuan Electric Company $>$ Henan Zhongtian Electrical Limited by Share Ltd $>$ Henan Wanli Transportation Company $>$ Elevator.

\section{Conclusions and Future Studies}

A method that can differentiate relevance indices is defined in this paper. Also, improved Deng's relational degree is introduced, and we prove that it distinguishes between strong and weak relevance indexes with greater precision. Then, according to new Deng's relational degree, we constructed a dynamic multiple-attribute decision-making model and applied it to the evaluation of private enterprises. The results verify the feasibility of the new decision-making model.

Future studies can be performed, based on the ideas in this paper. For example, we merely proposed an improved grey relational model, based on Deng's relational degree; other types of grey relational models, such as $T$ 's grey relational model, can be improved using exponential functions. The improved grey relational model can also be combined with clustering methods and decision-making methods. Thus, more applications of decision-making models that are based on the improved grey relational model can be identified. In terms of applications, all types of models 
should be improved and combined with regard to practical problems.

\section{Conflict of Interests}

The authors declare that there is no conflict of interests regarding the publication of this paper.

\section{Acknowledgments}

The authors would like to express their gratitude to the editor and anonymous reviewers for their helpful comments which improve the quality of the paper. The paper is sponsored by National Natural Science Foundation of China (71071077, 71371098); Fundamental Research Funds for the Central Universities (NC2012001, NZ2010006); Funding of Jiangsu Base of Major Projects on Philosophy and Social Sciences (2012JDXM005); National Social Sciences Program Fund (14ZDB151); Public Science and Technology Research Funds Projects of Ocean (201205038, 2013418034, and 201405029); and Humanities and Social Sciences Program of Ministry of Education (13JBGP005).

\section{References}

[1] N. Bryson and A. Mobolurin, "An action learning evaluation procedure for multiple criteria decision making problems," European Journal of Operational Research, vol. 96, no. 2, pp. 379386, 1997.

[2] S. B. Eom, "The intelligent development and structure of decision support system," Omega-International Journal of Management Science, vol. 26, no. 5, pp. 639-657, 1998.

[3] G. R. Jahanshahloo, F. H. Lotfi, and M. Izadikhah, "An algorithmic method to extend TOPSIS for decision-making problems with interval data," Applied Mathematics and Computation, vol. 175, no. 2, pp. 1375-1384, 2006.

[4] Y.-G. Sun and Y.-G. Dang, "Improvement on grey T's correlation degree," System Engineering Theory and Practice, vol. 28, no. 4, pp. 135-139, 2008.

[5] H. Y. Du, H. X. Shi, S. F. Liu, and Z. G. Fang, "Study on the model of grey periodic incidence judged on slope," Chinese Journal of Management Science, vol. 18, no. 1, pp. 128-132, 2010.

[6] K. Guo and Q. S. Zhang, "Spectral clustering based on grey relational analysis," Systems Engineering: Theory \& Practice, vol. 30, no. 7, pp. 1260-1265, 2010.

[7] X. M. Li, K. W. Hipel, and Y. G. Dang, "An improved grey relational analysis approach for panel data clustering," Expert Systems with Applications, vol. 42, no. 23, pp. 9105-9116, 2015.

[8] X. M. Li, Y. G. Dang, and J. J. Wang, "Grey generation rate relational analysis model based on grey exponential law and its application," Control and Decision, vol. 30, no. 7, pp. 1245-1250, 2015.

[9] S.-F. Zhang and S.-Y. Liu, "A GRA-based intuitionistic fuzzy multi-criteria group decision making method for personnel selection," Expert Systems with Applications, vol. 38, no. 9, pp. 11401-11405, 2011.

[10] G.-W. Wei, "Gray relational analysis method for intuitionistic fuzzy multiple attribute decision making," Expert Systems with Applications, vol. 38, no. 9, pp. 11671-11677, 2011.
[11] J. J. Jin, C. M. Mi, W. X. Xu, Q. F. Wang, and H. W. Wei, “The maximum entropy empowerment model for evaluating index considering the expert evaluation information," Chinese Journal of Management Science, vol. 20, no. 2, pp. 135-143, 2012.

[12] C. Li, K. J. Chen, and X. D. Xiang, "An integrated framework for effective safety management evaluation: application of an improved grey clustering measurement," Expert Systems with Applications, vol. 42, no. 13, pp. 5541-5553, 2015.

[13] Y.-H. Wang and Y.-G. Dang, "Method of grey fixed weight clustering comprehensive ex-post evaluation based on D-S evidential theory," System Engineering Theory and Practice, vol. 29, no. 11, pp. 128-134, 2009.

[14] J. H. Jung and W. T. Kwon, "Optimization of EDM process for multiple performance characteristics using Taguchi method and Grey relational analysis," Journal of Mechanical Science and Technology, vol. 24, no. 5, pp. 1083-1090, 2010.

[15] H. Wong and B. Q. Hu, "Application of interval clustering approach to water quality evaluation," Journal of Hydrology, vol. 491, no. 13, pp. 1-12, 2013.

[16] R. Rajesh and V. Ravi, "Supplier selection in resilient supply chains: a grey relational analysis approach," Journal of Cleaner Production, vol. 86, no. 15, pp. 343-359, 2015.

[17] J. Song, Y. G. Dang, and Z. M. Hua, "Study on group decisionmaking method based on grey cluster model," Control and Decision, vol. 25, no. 10, pp. 1593-1597, 2010.

[18] L. F. Hu, X. Guan, and Y. He, "A new approach for grey multiattribute decision making," Control and Decision, vol. 27, no. 6, pp. 895-898, 2012.

[19] X. X. Chen and S. F. Liu, "Study on grey multiple attribute group decision-making method without weight information," Chinese Journal of Management Science, vol. 16, no. 5, pp. 146-152, 2008.

[20] P.-D. Liu and Z.-L. Guan, "Research on multiple attribute decision-making under risk with continuous random variable and weight unknown," Systems Engineering and Electronics, vol. 31, no. 9, pp. 2133-2150, 2009.

[21] B. C. Yang and Y. Chen, "Grey relational decision-making model based on variable weight and TOPSIS method," Systems Engineering, vol. 29, no. 6, pp. 106-112, 2011.

[22] G.-W. Wei, "GRA method for multiple attribute decision making with incomplete weight information in intuitionistic fuzzy setting," Knowledge-Based Systems, vol. 23, no. 3, pp. 243-247, 2010.

[23] W.-S. Lee and Y.-C. Lin, "Evaluating and ranking energy performance of office buildings using Grey relational analysis," Energy, vol. 36, no. 5, pp. 2551-2556, 2011.

[24] M.-S. Kuo and G.-S. Liang, "Combining VIKOR with GRA techniques to evaluate service quality of airports under fuzzy environment," Expert Systems with Applications, vol. 38, no. 3, pp. 1304-1312, 2011.

[25] A. Samvedi, V. Jain, and F. T. S. Chan, "An integrated approach for machine tool selection using fuzzy analytical hierarchy process and grey relational analysis," International Journal of Production Research, vol. 50, no. 12, pp. 3211-3221, 2012.

[26] Y. Wang, "Analysis of FDI and Chinese regional economic discrepancy using grey incidence," Systems Engineering: Theory \& Practice, vol. 30, no. 3, pp. 426-430, 2010.

[27] H. Y. Wei, L. Zhang, Y. M. Liang, and Z. W. Yao, "Research on the effect of multidimensional interaction on service brand equitybased gray relational analysis," Journal of Management Sciences in China, vol. 14, no. 10, pp. 43-53, 2010. 
[28] J. Lin, H. Chiang, and C. C. Lin, "Tuning PID control parameters for micro-piezo-stage by using grey relational analysis," Expert Systems with Applications, vol. 38, no. 11, pp. 13924-13932, 2011.

[29] Z. Ma, H. Wang, A. Wu, G. Zeng, and X. Tu, "An intelligent decision support system for residential energy consumption and renewable energy utilization in rural China," Energy Sources B: Economics, Planning and Policy, vol. 9, no. 4, pp. 374-382, 2014.

[30] Y. M. Wang, G. S. Chen, and Z. L. Yan, "Law of annual maximum peak discharge at upper stream of the Yellow River," Journal of Water Resources \& Water Engineering, vol. 24, no. 2, pp. 80-82, 2013.

[31] Z. Liu, Y.-G. Dang, W.-Y. Qian, and W.-J. Zhou, "Grey grid incidence model based on panel data," Systems EngineeringTheory \& Practice, vol. 34, no. 4, pp. 991-996, 2014.

[32] G. Z. Feng and Y. H. Yi, "Evaluation of core competence of retail enterprise based on financial perspective," Management World, no. 12, pp. 52-55, 2012.

[33] E. Qi and F. Xia, "Evaluation research of the competitiveness of small and medium-sized enterprises based on overall evaluation," Journal of Dalian University of Technology, vol. 33, no. 2, pp. 76-80, 2012.

[34] Z. B. Feng and Y. C. Diao, "Comprehensive evaluation research of competitiveness of China life insurance enterprises," Journal of Shandong Academy of Governance, no. 1, pp. 65-71, 2013.

[35] X. Wang, L. Li, and H. X. Deng, "Comprehensive evaluation research of the competitiveness in China's regional construction industry-based on PP-DEA model," Technoeconomics \& Management Research, no. 8, pp. 23-28, 2013.

[36] F. T. Liu, S. H. Chen, and C. C. Gao, "Research on the competitiveness evaluation model of fourth party logistics enterprises based on AHP-Delphi method," Journal of Industrial Technological Economics, no. 2, pp. 83-88, 2011.

[37] L. Wang, "Construction of index system for evaluation of economic competitiveness of Hangzhou headquarters," Management and Administration, no. 11, pp. 130-131, 2013.

[38] L. X. Cai, "Empirical analysis of Xiamen's competitiveness of manufacturing industries," Special Zone Economy, no. 11, pp. 198-199, 2013.

[39] J. J. Zhang, "Evaluation of competitiveness of small and micro innovative enterprises," Statistics and Decision, no. 22, pp. 174176, 2013.

[40] F. Yang and B. Y. Zhu, "Construction of evaluation system for competitiveness of China's iron and steel enterprises," Statistics and Decision, no. 13, pp. 60-63, 2013.

[41] Y. Chang, Core competitiveness evaluation and promotion strategy of Z logistics company based on the AHP-TOPSIS [M.S. thesis], South China University of Technology, Guangzhou, China, 2012.

[42] N. M. Xie, Research on the Methods of Grey Systems Modeling, Nanjing University of Aeronautics and Astronautics, 2008.

[43] S. F. Liu, Y. G. Dang, and Z. G. Fang, Grey Systems: Theory and Application, Science Press, Beijing, China, 2004. 


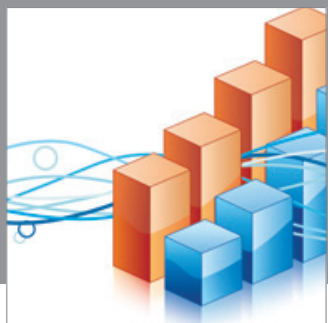

Advances in

Operations Research

mansans

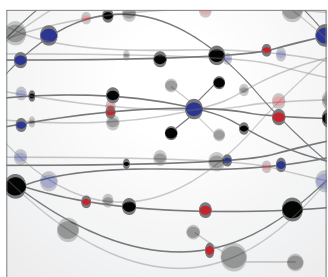

The Scientific World Journal
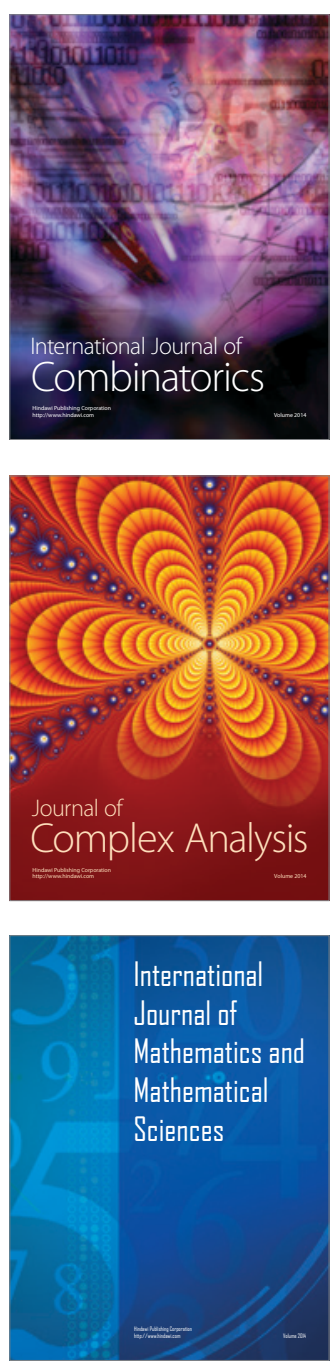
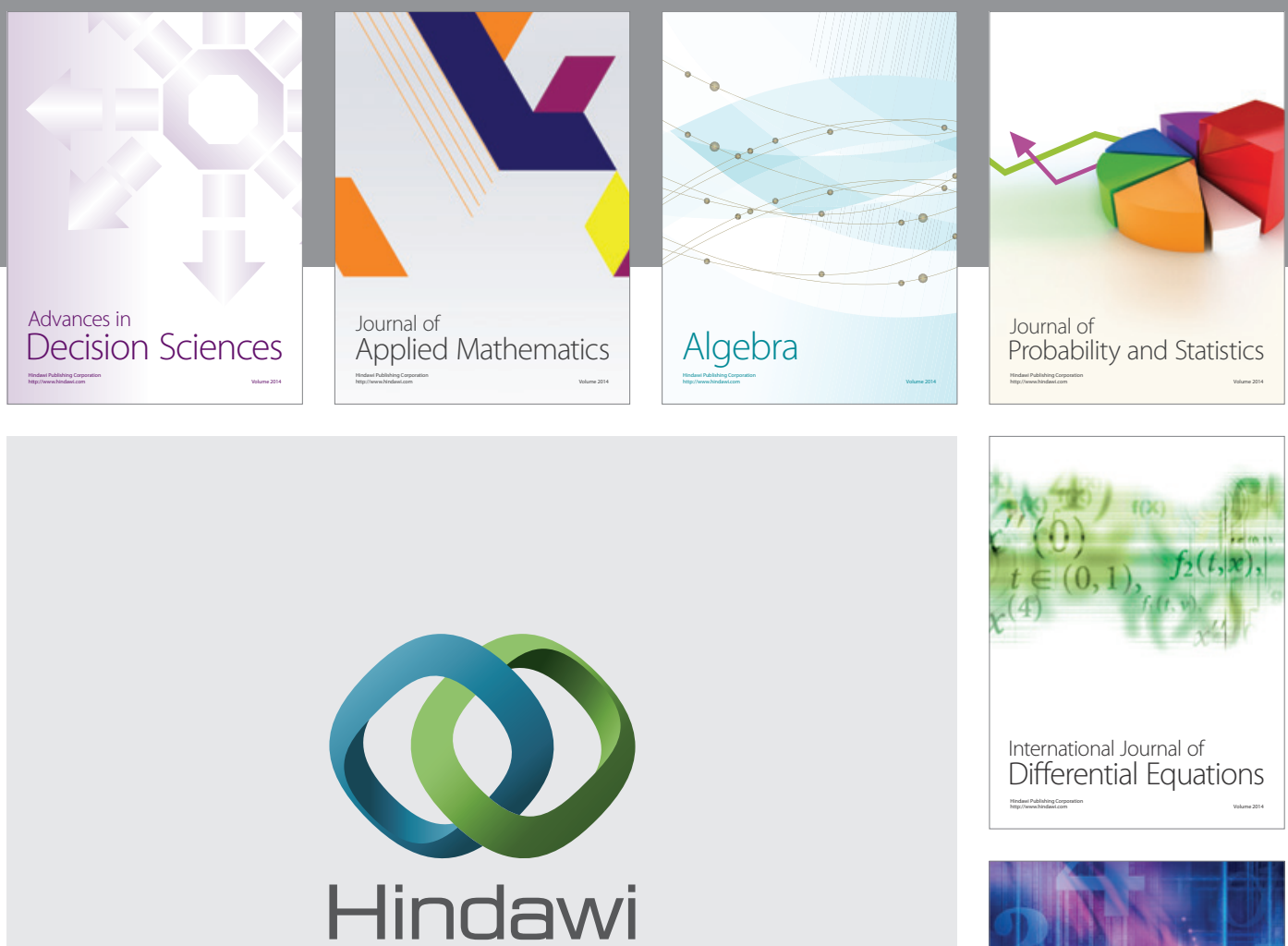

Submit your manuscripts at http://www.hindawi.com
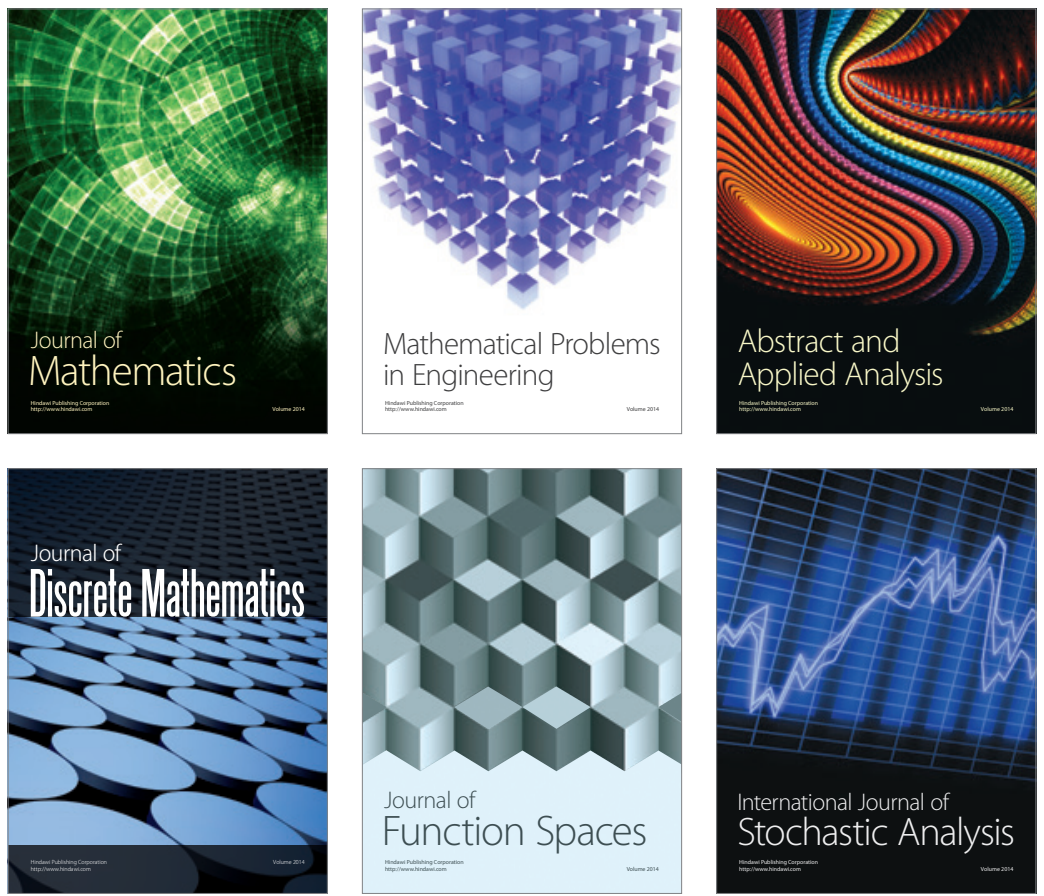

Journal of

Function Spaces

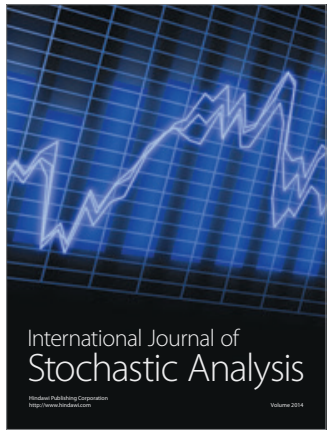

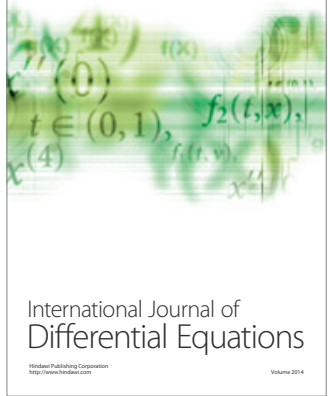
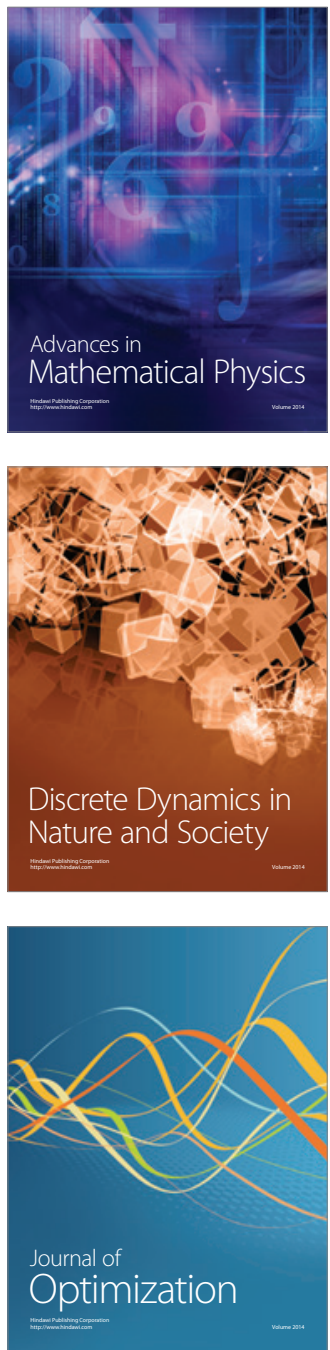